\title{
The Tol1 element of the medaka fish, a member of the $h A T$ transposable element family, jumps in Caenorhabditis elegans
}

\author{
K Kodama, S Takagi and A Koga \\ Division of Biological Science, Graduate School of Science, Nagoya University, Nagoya, Japan
}

\begin{abstract}
Tol1 is a DNA-based transposable element residing in the genome of the medaka fish Oryzias latipes, and has been proven to be transposed in various vertebrate species, including mammals. This element belongs to the hAT (hobol Activator/Tam3) transposable element family, whose members are distributed in a wide range of organisms. It is thus possible that Tol1 is mobile in organisms other than vertebrates. We here show that transposition of this element occurs in the nematode Caenorhabditis elegans. A donor plasmid containing a Tol1 element and a helper plasmid carrying the transposase gene were delivered into gonad cells and, after several generations of culturing, were recovered from worms. PCR analysis of the donor plasmid, using primers that encompassed the Tol1 element, revealed
\end{abstract}

excision of the Tol1 portion from the plasmid. Analysis of genomic DNA of the worms by the inverse PCR method provided evidence that Tol1 had been integrated into the C. elegans chromosomes. Vertebrates and $C$. elegans are phylogenetically distantly related organisms in that the former are deuterostomes and the latter a protostome animal. Our results indicate (1) the transposition reaction of the Tol1 element requires, besides the transposase, no factors from host cells, or (2) the host factors, even if required, are those that are common to protostomes and deuterostomes. The results also have significance for the development of a gene transfer vector and other biotechnology tools for $C$. elegans. Heredity (2008) 101, 222-227; doi:10.1038/hdy.2008.47; published online 28 May 2008

Keywords: transposition; C. elegans; $h A T$ family; host factors; evolution

\section{Introduction}

A large number of DNA-based transposable elements have been identified in various organisms, and many of them can be grouped into transposable element families, based mainly on the overall structures and amino-acid sequence similarities of internal genes. Ranges of host organisms differ among families, and dependence of the transposition reaction on host species-encoded factors is considered to be one determinant of the host ranges (cf. Lozovskaya et al., 1995). For example, the $P$ element of Drosophila melanogaster is prevalent in this species, but absent in its sibling species $D$. simulans. Kimura and Kidwell (1994) initiated experimental populations of these two species containing similar copy numbers of the element, and maintained them under similar environmental conditions, observing a higher copy number in D. melanogaster in subsequent generations. As the authors concluded, some host factors would be the most likely explanation for the observed difference.

Of several transposable element families known to date, the mariner/Tc1 family is most conspicuous in that member elements are distributed in a wide range of organisms. The mariner and Tc1 elements were found in

Correspondence: $\operatorname{Dr}$ A Koga, Division of Biological Science, Graduate School of Science, Nagoya University, Nagoya 464-8602, Japan.

E-mail: koga@bio.nagoya-u.ac.jp

Received 20 January 2008; revised 27 March 2008; accepted 30 April 2008; published online 28 May 2008
D. mauritiana (Jacobson et al., 1986) and the nematode Caenorhabditis elegans (Emmons et al., 1983), respectively, and related elements are known to occur in diverse taxa, including planarians (Garcia-Fernandez et al., 1995), annelids (Jee et al., 2007), vertebrates (Smit and Riggs, 1996), plants (Jarvik and Lark, 1998) and fungi (Langin et al., 1995). Manifestation of such a wide distribution leads to the inference that elements of this family require no or few factors produced from host cells for their transposition reaction. This has already been demonstrated with mariner, Tc1 and some other elements: transposition occurs in vitro as long as a purified transposase is provided (Vos et al., 1996; Lampe et al., 1996). It was also inferred and then proven that elements of this family move when they are artificially introduced into organisms other than their original host species. The most notable examples from the viewpoint of the host species distance are their transposition in vertebrates (Figure 1). These elements thus retain the transposition activity even when they cross the protostome/deuterostome boundary. Protostomes and deuterostomes are two main branches of commonly accepted phylogenetic trees for animals (Figure 1). The piggyBac element of another family has also been shown to be able to cross this boundary.

There is one other transposable element family whose members are broadly distributed across kingdoms. It is the $h A T$ family named for the hobo, Activator and Tam3 elements from D. melanogaster (McGinnis et al., 1983), the maize Zea mays (Fedoroff et al., 1983) and the snapdragon 


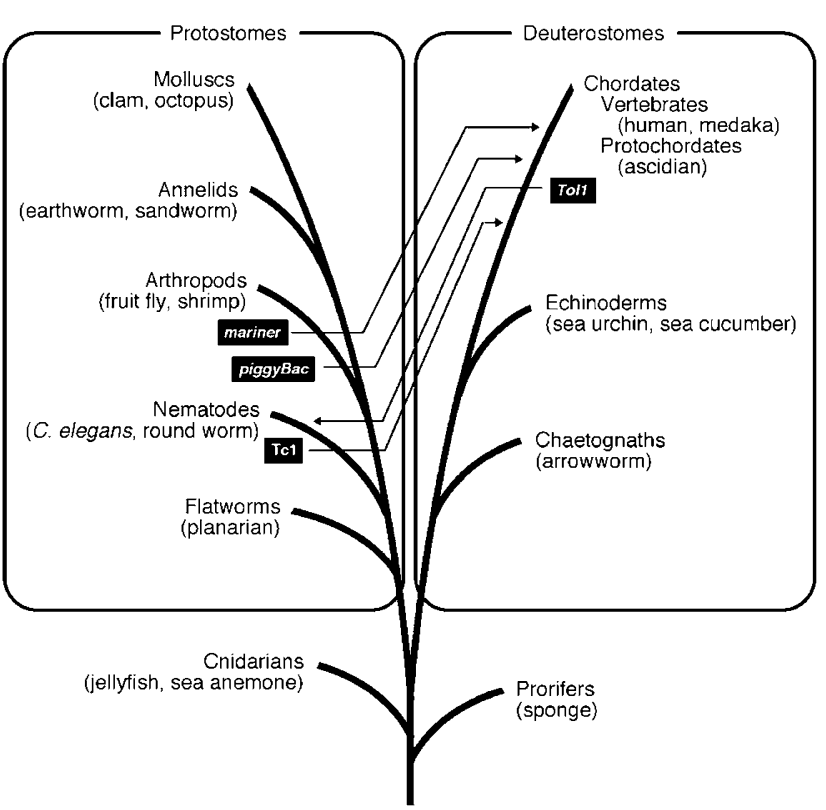

Figure 1 Commonly accepted phylogenetic tree for animals. Phyla were arbitrarily selected, and genetic or temporal distances are not reflected in the branch lengths. The items indicated in white letters are transposable elements that have been shown to be active across the protostome/deuterostome boundary. They were tested for transposition activity in organisms of the phyla indicated by the arrows. The recipient species were: mariner, zebrafish animals (Fadool et al., 1998); Tc1, human culture cells (Schouten et al., 1998); piggyBac, mouse animals (Ding et al., 2005); Tol1, C. elegans animals (present study)

Antirrhinum majus (Coen et al., 1986), respectively. Similar to the case of the mariner/Tc1 family, no or low dependence on host factors is inferred for elements of this family (Calvi et al., 1991), and this has been confirmed at least in plants (Kunze, 1996).

Tol1 is a DNA-based transposable element residing in the genome of the medaka fish Oryzias latipes (Koga et al., 1995). We have recently found a medaka fish strain that was highly mutable because of high-frequency transposition of the element, and identified an autonomous Tol1 copy by analyzing this strain (Koga et al., 2007a). The autonomous copy carried a transposase gene, and a BLAST search of its amino-acid sequence revealed that Tol1 is an hAT element (Koga et al., 2007b). Tol1 is mobile in many vertebrate species, including the medaka fish (Tsutsumi et al., 2006), zebrafish (manuscript under review), frog (Hikosaka and Koga, 2007), and human and mouse (Koga et al., 2007a). Vertebrates are located on the phylogenetic branch of deuterostomes. The purpose of this study was to examine if Toll can be transposed in a protostome animal. For this purpose, we first generated transgenic $C$. elegans lines bearing a nonautonomous Tol1 element and the transposase gene. We then analyzed worms for excision of the element from the donor plasmid and reintegration into the C. elegans chromosomes. The results clearly indicated the occurrence of both in C. elegans cells. Thus, the Tol1 element is mobile in both protostomes and deuterostomes. Our results thus suggest that no factors are required from host cells, or that the host factors, even if required, are those that are common to protostomes and deuterostomes.

\section{Materials and methods}

\section{C. elegans breeding}

The Bristol isolate $\mathrm{N} 2$ of $C$. elegans was used as a recipient of foreign DNAs. This strain was homozygous for the wild-type rol-6 allele. Nematode culture at $20^{\circ} \mathrm{C}$ and genetic manipulations used standard procedures (Brenner, 1974).

\section{Plasmid construction}

We prepared three kinds of plasmids: donor, helper and marker. The Tol1 element was expected to be excised from the donor plasmid and then integrated into C. elegans chromosomes. The helper plasmid had the role of providing the transposase enzyme that catalyzes the excision and insertion reactions. The marker plasmid was used to screen worms for those carrying the donor and helper plasmids after microinjection.

The donor plasmid is a clone of a 4.3-kb-long fragment of the medaka fish genomic DNA, which contains a 1.9kb-long Tol1 element and its flanking chromosomal regions (Figure 2). The 1.9-kb element corresponds to the $1.5-\mathrm{kb}$ left- and $0.4-\mathrm{kb}$ right-terminal regions of the 4.4-kb-long, autonomous Tol1 element, and most parts of the transposase gene are lacking (see Figure 2 of Koga et al., 2007a).

The helper plasmid was generated by cloning the coding region of the Tol1 transposase cDNA (Koga et al., 2007a) into the NheI site of pPD49.78. pPD49.78 contains the promoter region of the $C$. elegans heat-shock protein gene hsp16-2 (Stringham et al., 1992) and the 3'-terminal region of the $u n c-54$ gene, the NheI site being located between these.

The marker plasmid was pRF4 (Mello and Fire, 1995). This is a clone of the rol-6 (su1006) gene of C. elegans, which is dominant over the wild-type allele and causes a twisted body shape to its carrier.

\section{Generation of transgenic lines}

The helper plasmid (150 ng $\mu \mathrm{l}^{-1}$ ) and the donor plasmid $\left(15 n g \mu 1^{-1}\right)$ were mixed with the marker plasmid $\left(150 \mathrm{ng}^{-1}\right)$, and then injected into the gonad of hermaphrodites. DNAs introduced into C. elegans by this procedure are known to undergo intermolecular ligation to generate a large extrachromosomal array that contains many copies of the transgenic DNA, which can be partitioned during mitosis (Mello et al., 1991). The array can be expected to contain all three plasmids. Transgenic worms were selected based on the phenotype of the twisted body shape (roller). Two lines, ST1275 and ST1278, which continually segregated progeny exhibiting the roller phenotype, were established. PCR analyses confirmed that both lines contained both helper and donor plasmids (data not shown).

\section{Heat-shock induction of transposase}

For each line, two worm cultures were started by placing 10 young adult hermaphrodites exhibiting the roller phenotype on NGM plates seeded with a lawn of OP50 Escherichia coli. The two cultures are designated as $\mathrm{H}$ (heat-shock treated) and N (nontreated), hereafter. From $12 \mathrm{~h}$ after starting the cultures, animals of the $\mathrm{H}$ culture were heat-shocked by placing the plate in an air incubator set at $37^{\circ} \mathrm{C}$ for $30 \mathrm{~min}$ twice a day for 4 days. 

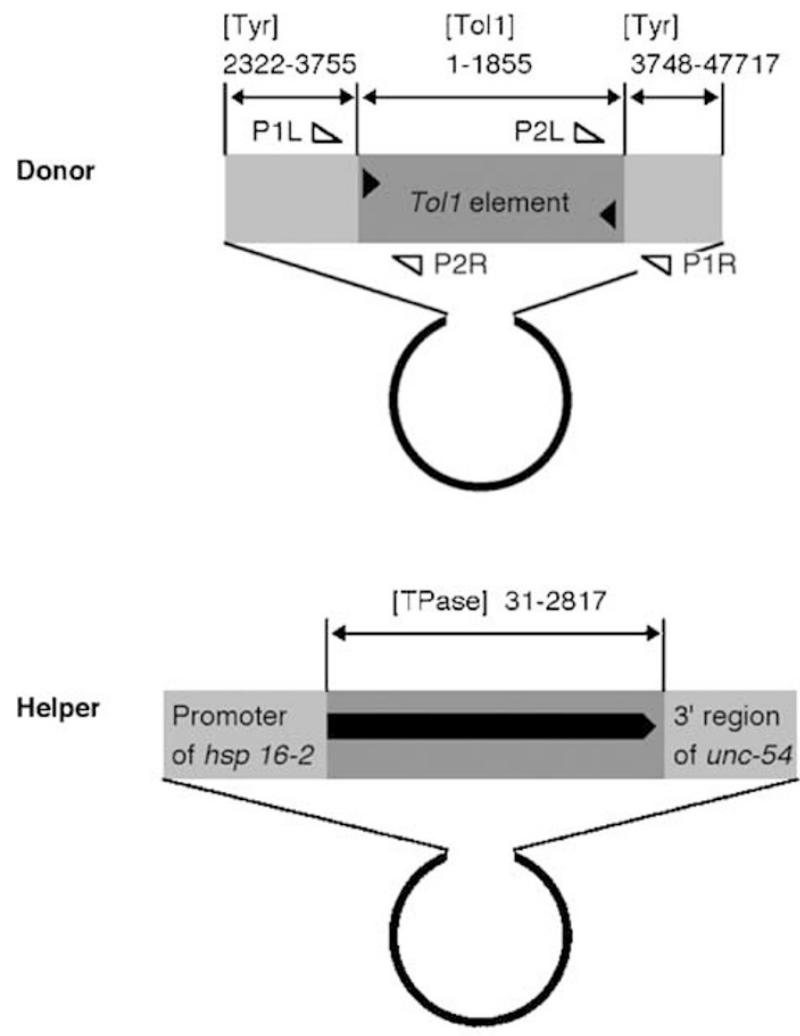

Marker

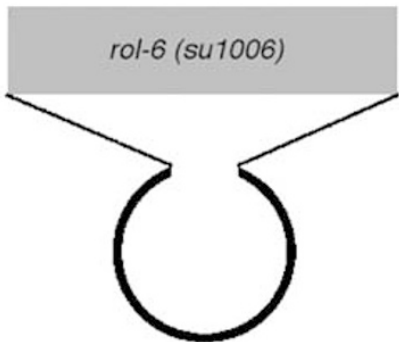

Figure 2 Organization of plasmids. Nucleotide blocks included as components of the plasmids are indicated with abbreviations for EMBL sequence files and the nucleotide positions. The abbreviations are: (Tyr) AB010101 (the tyrosinase gene of medaka), (Tol1) D42062 (a 1.9-kb-long copy of the Tol1 element) and (Tpase) AB264112 (cDNA for the transposase). The black triangles at the ends of the Tol1 element indicate terminal inverted repeats of $14 \mathrm{bp}$ The open triangles show locations and directions of the PCR primers. P1s are for detection of excision, and P2s for insertion.

During the heat-shock treatments, animals of the $\mathrm{N}$ culture were left in a $20^{\circ} \mathrm{C}$ incubator. The $\mathrm{N}$ culture had the role of a negative control for the transposase function.

At $12 \mathrm{~h}$ after the final heat-shock treatment, animals were collected by washing the plates with distilled water.

Analysis of donor plasmid for excision of Tol1

DNA was extracted from the worms with the Puregene DNA purification kit (Gentra Systems Inc., Minneapolis, MN, USA). Using these DNA samples as a template, we conducted a PCR analysis of the donor plasmid using the ExTaq polymerase (Takara Bio Inc., Otsu, Japan) for excision of the Tol1 element. The primers used were P1L

(nt 3594-3623 of EMBL file AB010101) and P1R (nt 38953866). The distance between these two primers on the donor plasmid was $2.2 \mathrm{~kb}$. Precise excision of the Tol1 portion, which is $1.9 \mathrm{~kb}$ in length, from the donor plasmid was expected to result in a PCR product of $0.3 \mathrm{~kb}$. The PCR conditions were as follows: $\left(94^{\circ} \mathrm{C}, 120 \mathrm{~s}\right)$, $46 \times\left(94{ }^{\circ} \mathrm{C}, 20 \mathrm{~s} ; 64{ }^{\circ} \mathrm{C}, 20 \mathrm{~s} ; 72{ }^{\circ} \mathrm{C}, 15 \mathrm{~s}\right),\left(72{ }^{\circ} \mathrm{C}, 60 \mathrm{~s}\right)$.

\section{Analysis of genomic DNA for insertion of Tol1}

We used the inverse PCR technique to detect insertion. As the first step, we digested $4.0 \mu \mathrm{g}$ of DNA with BamHI, EcoRI or XbaI and NheI. Because the Tol1 portion of the donor plasmid did not contain cutting sites for these restriction enzymes, the digestion treatment was expected to yield restriction fragments consisting of the entire Tol1 element and its flanking chromosomal regions. We electrophoresed the digested DNAs on a $1.0 \%$ agarose gel, cut out a gel piece containing $1.9-6.0 \mathrm{~kb}$ DNA fragments and recovered the fragments from the gel piece. This was to exclude fragments of $<1.9 \mathrm{~kb}$ that were too small to contain the entire Tol1 element and those of $>6.0 \mathrm{~kb}$ that included fragments too long to be amplified by inverse PCR.

Size-selected DNA fragments were ligated with T4 DNA ligase, concentrated through ethanol precipitation, and then used as a template for inverse PCR. The primers for this PCR analysis were P2L (nt 1758-1787 of EMBL file D42062) and P2R (nt 130-101). The PCR conditions were as follows: $\left(94^{\circ} \mathrm{C}, 120 \mathrm{~s}\right), 40 \times\left(94{ }^{\circ} \mathrm{C}, 20 \mathrm{~s}\right.$; $\left.64{ }^{\circ} \mathrm{C}, 20 \mathrm{~s} ; 72{ }^{\circ} \mathrm{C}, 150 \mathrm{~s}\right),\left(72{ }^{\circ} \mathrm{C}, 180 \mathrm{~s}\right)$. The obtained PCR products were cloned into pT7Blue-2 (Takara Bio Inc.) and sequenced.

Insertion sites of Tol1 elements in the C. elegans genome were determined by using C. elegans Blast Server (http:/ / www.sanger.ac.uk/cgi-bin/blast/submitblast/c_elegans).

\section{Results}

\section{Detection of excision by PCR}

We conducted a PCR analysis of DNAs extracted from worms bearing transgenes containing the donor plasmid, and the reaction mixtures were electrophoresed on an agarose gel. With both lines, the DNA sample from the $\mathrm{H}$ culture produced DNA fragments of about $0.3 \mathrm{~kb}$, and no PCR product was observed in the lane for the $\mathrm{N}$ culture (data not shown). It was thus suggested that excision of the Tol1 element occurred in worms of the $\mathrm{H}$ cultures. The results do not necessarily mean that excision did not occur in worms of the $\mathrm{N}$ cultures, but the frequency was, even if excision occurred, lower than in worms of the $\mathrm{H}$ cultures.

\section{Confirmation of excision by sequencing}

To determine the breakpoints of the rearrangements of the donor plasmid, we cloned PCR products from the ST1275-H and ST1278-H cultures. Several clones were available from each line, but only one clone was chosen at random. The two clones were then sequenced along both strands (Figure 3). In both clones, the entire Tol1 sequence was found to have been removed and parts of the target site duplication (TSD) sequences had been left behind. In addition, 8-bp- and 15-bp-long sequences had been inserted at the breakpoints. These extra nucleotides 


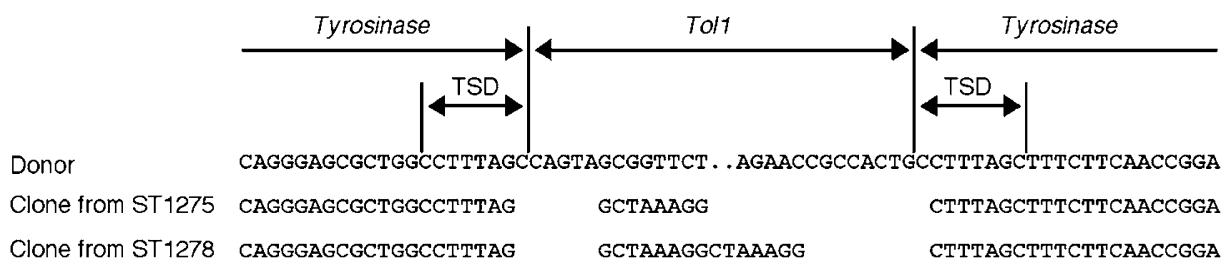

Figure 3 Nucleotide sequences of excision breakpoints and their vicinities. The top line is the sequence of the donor plasmid, shown for reference. The other two lines are the sequences of cloned PCR products. Dots indicate there are nucleotides not shown here.

appear to be unrelated to the Tol1 sequence, and consist of sequences complementary to the TSD sequence.

The deletion of the entire Tol1 region provides clear evidence for the occurrence of excision. The excision was not strictly precise in both samples, as has commonly been observed with Tol1 (Koga et al., 2007b; Hikosaka and Koga, 2007).

\section{Detection of insertion by inverse PCR}

DNAs extracted from worms of the $\mathrm{H}$ cultures were digested with restriction enzymes, ligated with T4 DNA ligase and then used as templates for inverse PCR analysis. Several PCR products of different sizes were observed in single lanes, suggesting that the DNA samples contained more than one insertion at different chromosomal locations (data not shown). Worms of the $\mathrm{N}$ cultures were not included in this analysis because our purpose was to demonstrate the occurrence of insertion and this was likely to be attained with only worms of the $\mathrm{H}$ cultures.

\section{Confirmation of insertion by sequencing}

The PCR products of each lane were cloned into plasmids and sequenced. Of four clones examined, one appeared to originate from the donor plasmid integrated into the extrachromosomal array because the sequences of the Tol1-flanking regions were identical to those of the donor plasmid. The other three clones were found to have Tol1-flanking regions whose sequences were different from those of the donor plasmid (Figure 4). A BLAST search against the $C$. elegans nucleotide sequence database revealed that the left- and right-flanking regions were adjacently located in single sequence files. The sequences of clones $a, b$ and $c$ were found to be included in the sequences of the fosmid clone designated as H10E21 (tip of chromosome 3, nucleotide identity $100 \%$ ), cosmid clone T19B10 (one-third of the chromosome length from one end of chromosome 4, identity 98\%) and cosmid clone F19B6 (middle of chromosome 5, identity 99\%), respectively. No coding sequence was predicted at their insertion sites. In clones a and b, 8-bp sequences on the left side and the right side of the Tol1 element were identical, which indicated that TSD characteristic of insertion of the Tol1 transposition in the medaka fish genome also took place in C. elegans. Clone c did not have an apparent TSD.

The above findings that the Tol1 element was inserted in three different $C$. elegans genomic sequences indicate that the Tol1 element had been inserted into the chromosomes by transposition.

\section{Discussion}

\section{Transposition of Tol1 in a protostome}

In the evolutionary stage of acquisition of triploblastic embryogenesis by diploblastic metazoans, they diverged into the two branches of protostomes and deuterostomes. In the present study, we have demonstrated that the Tol1 element native to a deuterostome animal (medaka fish) is transposed in cells of a protostome animal (C. elegans). Our results indicate that host-cell-encoded factors indispensable for the transposition reaction of the Toll element are, even if required, those that are common to protostomes and deuterostomes. This could be helpful in explaining why $h A T$ elements are distributed in a wide range of organisms, including both protostome and deuterostome animals. We have previously shown evidence for horizontal (interspecific) transfer of Tol2, another $h A T$ element of the medaka fish, between two species of the genus Oryzias (Koga et al., 2000). The present results support the view that horizontal transfer of $h A T$ elements might occur between more distantly related animals.

\section{Transmission of insertions across generations}

The results of the inverse PCR analysis suggest that Tol1 elements inserted in the $C$. elegans chromosomes were transmitted across generations.

Electrophoresis of the inverse PCR products exhibited distinct bands in the present study. We have previously applied exactly the same procedures to human culture cells (Koga et al., 2007b). On conducting inverse PCR with cells after proliferation through several rounds of cell division, we observed smeared PCR products. This result indicates that there were a great number of insertions of different origins, each being low in frequency. We then prepared cell clones from single cells. Inverse PCR with these cells, in contrast, resulted in a few distinct bands in electrophoresis photos. These results suggest that in the present study the Toll insertions passed through a bottleneck after their occurrence. Such a bottleneck is likely to have arisen through changes in generations. A possible scenario is as follows: insertions that occurred in germ-line cells and/ or undifferentiated blastomeres were transmitted to subsequent generations through germ cells, and amplified in somatic cells of the animals used for DNA preparation.

\section{Possibility of transposition in other taxa}

The findings that the Tol1 element is transposed in chordate and nematode phyla suggest the mobility of this element in other animals located at phylogenetic 


\begin{abstract}
Donor GGGGCCCTGTCTGGTCGCGGGTTCTGTGCAGACGTCTCGGTTTCAGAC
Clone a CATAAAACCTGGTGCTAAACACGATAAACTTATTGAAACATGGCACAC

Clone $b$ TGATACAGAATTAGCAGAAACTACAGGGGCCCTGTTTCTCTCGGGTCT

Clone $c$ AATAGAGTTAAAGATTTTGATTTCAAACTTCCATAGTAGACAGGCATA
\end{abstract}

GAGCCCAACGGGCCGCAGTACCCTCACAGCGGGATCGATGACAGGGAG ACCTGAAATTTTCCGATTTTGAAAAAGTGCTCAGCAGGTACCCCCAGC TTGCACACTTTTTTCAAAAAAGGGGTTCTTAGGGTCTCGCAATTCTAT TCTTTATGCCTACCTAGGCGCAGTCTACTTTTTGGTTTTGGTGTGGTG

CGCTGGCCTTTAGCCAGTAGCGGTTCTAGGCACGGGCCGTCCGG.... TGGACCACTGGGGTCAGTAGCGGTTCTAGGCACGGGCCGTCCGG.... CTAGATCTGGGAAACAGTAGCGGTTCTAGGCACGGGCCGTCCGG.... TTAGGTTGATCTTTCCGTAGCGGTTCTAGGCACGGGCCGTCGG. .

.... GGGGAGTAATTCAGGGTAGAACCGCCACTGCCTTTAGCTTTCTT
.... GGGGAGTAATTCAGGGTAGAACCGCCACTGACTGGGGTGTCCAG
.... GGGGAGTAATTCAGGGTAGAACCGCCCCTGCTGGGAAATTTCAG
... GGGGAGTAATTCAGGGTAGAACCGCCACTGATCACCACCCAGTA

CAACCGGACGTGTCGTTGTGCAGGAAACTATGGAGGGTTTAACTGTGG AAGGGTACCCCGTTATATTGGAAAATTTGGGCCCTCTGTCATGTTTAA TAATACCTGAAAACCAAATAACCTAGGAATATTGGGAGACGTTGTCCA TAAAACCACTGAAAATTACGTACCAACAACGTTATTTTTCTAAACAAA

GGAATGCAGATTCGGTTACTGGGGCTCCAACTGTGCTGAGTACAGAGA
CCAATTTTTTGAATTCCAAGACCCTAAATTTTTCCAATTTTTACC
AGAAGAAAAGTTTTGGCATTTCAGAAATAGGTAAAATATTTCCAAAAT
AAACAACAACGTTATTTTTCTAAATAACTGCATGAACAATAAATGAAT

Figure 4 Nucleotide sequences around insertion sites. The top line is the sequence of the donor plasmid, shown for reference. The other three lines are sequences of clones of three different inverse PCR products. Clones a and b originate from PCR reactions with ST1275/EcoRI, and clone c from ST1275/BamHI. The black and gray shaded regions are the Tol1 terminal regions and the target site duplications (TSDs), respectively. Clone c does not have an apparent TSD.

positions closer to the origin of animals, including echinoderms, flatworms and even diploblastic animals, such as cnidarians. Moreover, it is inferable that the element is mobile in other higher organisms, such as arthropods and molluscs (Figure 1). If this is proven, we can hold the view that the independence of $h A T$ family elements from host-species-specific factor(s) is as low as that of mariner/Tc1 family elements.

\section{Significance in biotechnology applications}

Transposable elements are used as powerful tools for basic and translational research. Applications include gene transfer, mutagenesis and gene/promoter/enhancer trapping. In C. elegans, the Tc1 element, which was identified originally in this species, is transposed efficiently, and has been utilized for insertion mutagenesis (Plasterk and van Luenen, 1997; Bessereau, 2006). Using native Tc transposons, including the Tc1 element, as mutagens, however, has some drawbacks: mobilization of Tc transposons is not restricted to a single class of elements. In addition, the $C$. elegans genome harbors plenty of its copies that complicate the identification of the mutagenic insertion. Using exogenous transposon systems would be a way to circumvent these limitations. Indeed, the mariner element isolated from $D$. mauritiana has been demonstrated to be mobile in C. elegans (Bessereau et al., 2001), and has served as a useful tool for tagged mutagenesis.

The Tol1 element has already been developed as a gene transfer vector for vertebrates (Koga et al., 2007a). Our present results on its mobility in C. elegans have paved the way for the development of a superior gene transfer vector and other genetic tools for this useful model organism. Considering the possible mobility of this element in a wide range of animals, technologies already developed for vertebrates and those to be developed for C. elegans might be directly applicable to various animals.

\section{Acknowledgements}

We thank Andrew Fire for providing the plasmid pPD49.78, and Hiroshi Hori for helpful discussion. This work was supported by grant nos. 18657069 (to ST) and 19570003 (to AK) from the MEXT of Japan, and a grant from the Yamada Science Foundation (to AK).

\section{References}

Bessereau JL (2006). Transposons in C. elegans. In: The C. elegans Research Community (ed). WormBook, doi/10.1895/wormbook.1.70.1 [http://www.wormbook.org/]. pp 1-13. 
Bessereau JL, Wright A, Williams DC, Schuske K, Davis MW, Jorgensen EM (2001). Mobilization of a Drosophila transposon in the Caenorhabditis elegans germ line. Nature 413: 70-74.

Brenner S (1974). The genetics of Caenorhabditis elegans. Genetics 77: 71-94.

Calvi BR, Hong TJ, Findley SD, Gelbart WM (1991). Evidence for a common evolutionary origin of inverted repeat transposons in Drosophila and plants: hobo, Activator, and Tam3. Cell 66: 465-471.

Coen ES, Carpenter R, Martin C (1986). Transposable elements generate novel spatial patterns of gene expression in Antirrhinum majus. Cell 47: 285-296.

Ding S, Wu X, Li G, Han M, Zhuang Y, Xu T (2005). Efficient transposition of the piggyBac (PB) transposon in mammalian cells and mice. Cell 122: 473-483.

Emmons SW, Yesner L, Ruan KS, Katzenberg D (1983). Evidence for a transposon in Caenorhabditis elegans. Cell 32: 55-65.

Fadool JM, Hartl DL, Dowling JE (1998). Transposition of the mariner element from Drosophila mauritiana in zebrafish. Proc Natl Acad Sci USA 95: 5182-5186.

Fedoroff N, Wessler S, Shure M (1983). Isolation of the transposable maize controlling elements Ac and Ds. Cell 35: 235-242.

Garcia-Fernandez L, Bayascas-Ramirez JR, Marfany G, MunozMarmol AM, Casali A, Baguna J et al. (1995). High copy number of highly similar mariner-like transposons in planarian (Platyhelminthe): evidence for a trans-phyla horizontal transfer. Mol Biol Evol 12: 421-431.

Hikosaka A, Koga A (2007). PCR detection of excision suggests mobility of the medaka fish Tol1 transposable element in the frog Xenopus laevis. Genet Res 89: 201-206.

Jacobson JW, Medhora MM, Hartl DL (1986). Molecular structure of a somatically unstable transposable element in Drosophila. Proc Natl Acad Sci USA 83: 8684-8688.

Jarvik T, Lark KG (1998). Characterization of Soymar1, a mariner element in soybean. Genetics 149: 1569-1574.

Jee SH, Kim GE, Hong SH, Seo SB, Shim JK, Park SC et al. (2007). Characterization of EamaT1, a member of maT family of transposable elements from the earthworm Eisenia andrei (Annelida, Oligochaeta). Mol Genet Genom 278: 479-486.

Kimura K, Kidwell MG (1994). Differences in $P$ element population dynamics between the sibling species Drosophila melanogaster and Drosophila simulans. Genet Res 63: 27-38.

Koga A, Higashide I, Hori H, Wakamatsu Y, Kyono-Hamaguchi Y, Hamaguchi S (2007b). The Tol1 element of medaka fish is transposed with only terminal regions and can deliver large DNA fragments into the chromosomes. J Hum Genet 52: 1026-1030.

Koga A, Inagaki H, Bessho Y, Hori H (1995). Insertion of a novel transposable element in the tyrosinase gene is responsible for an albino mutation in the medaka fish, Oryzias latipes. Mol Gen Genet 249: 400-405.
Koga A, Shimada A, Kuroki T, Hori H, Kusumi J, KyonoHamaguchi Y et al. (2007a). The Tol1 transposable element of the medaka fish moves in human and mouse cells. J Hum Genet 52: 628-635.

Koga A, Shimada A, Shima A, Sakaizumi M, Tachida H, Hori H (2000). Evidence for recent invasion of the medaka fish genome by the Tol2 transposable element. Genetics 155: 273-281.

Kunze R (1996). The maize transposable element activator (Ac). In: Saedler H, Gierl A (eds). Curr Top Microbiol Immunol 204 161-194.

Lampe DJ, Churchill ME, Robertson HM (1996). A purified mariner transposase is sufficient to mediate transposition in vitro. EMBO J 15: 5470-5479.

Langin T, Capy P, Daboussi MJ (1995). The transposable element impala, a fungal member of the Tc1-mariner superfamily. Mol Gen Genet 246: 19-28.

Lozovskaya ER, Hartl DL, Petrov DA (1995). Genomic regulation of transposable elements in Drosophila. Curr Opin Genet Dev 5: 768-773.

McGinnis W, Shermoen AW, Beckendorf SK (1983). A transposable element inserted just $5^{\prime}$ to a Drosophila glue protein gene alters gene expression and chromatin structure. Cell 34: 75-84.

Mello C, Fire A (1995). DNA transformation. Methods Cell Biol 48: 451-482.

Mello CC, Kramer JM, Stinchcomb D, Ambros V (1991). Efficient gene transfer in C. elegans: extrachromosomal maintenance and integration of transforming sequences. EMBO J 10: 3959-3970.

Plasterk RHA, van Luenen HG (1997). Transposons. In: Riddle DL, Blumenthal T, Meyer BJ, Priess JR (eds). C. elegans II. Cold Spring Harbor Laboratory Press: Plainview, New York, pp 97-116.

Schouten GJ, van Luenen HG, Verra NC, Valerio D, Plasterk RH (1998). Transposon Tc1 of the nematode Caenorhabditis elegans jumps in human cells. Nucleic Acids Res 26: 3013-3017.

Smit AF, Riggs AD (1996). Tiggers and DNA transposon fossils in the human genome. Proc Natl Acad Sci USA 93: 1443-1448.

Stringham EG, Dixon DK, Jones D, Candido EP (1992). Temporal and spatial expression patterns of the small heat shock (hsp16) genes in transgenic Caenorhabditis elegans. Mol Biol Cell 3: 221-233.

Tsutsumi M, Imai S, Kyono-Hamaguchi Y, Hamaguchi S, Koga A, Hori H (2006). Color reversion of the albino medaka fish associated with spontaneous somatic excision of the Tol1 transposable element from the tyrosinase gene. Pigment Cell Res 19: 243-247.

Vos JC, De Baere I, Plasterk RH (1996). Transposase is the only nematode protein required for in vitro transposition of Tc1. Genes Dev 10: 755-761. 